\title{
Non-Parameningeal Head and Neck Area
}

National Cancer Institute

\section{Source}

National Cancer Institute. Non-Parameningeal Head and Neck Area. NCI Thesaurus. Code C91069.

The superficial areas of head and neck including the oral cavity, buccal mucosa, and the parotid region. 